\section{INTENSE NEURALGIC PAIN IN THE ARMS AFTER CHILDBIRTH.}

BY A. C. GEDDES, M.D. EDIN., PROFESSOR OF AYATOMY, ROYAT COLLEGE OF SURGEONS IN IRFLAND.

THIS note is published in the hope that the facts it records may prove helpful to some practitioner. The writer is aware that in publishing it he is trespassing in fields with which he has no direct professional concern, and for this he apologises.

Eighteen months ago (autumn, 1910) the writer was asked by a medical colleague, Dr. $A$, if he could suggest any anatomical explanation for the occurrence of intense neuralgic pains in the arms after childbirth. Dr. A said that he had met with the condition once or twice in the course of practice, and that his wife, who had given birth to an unusually large boy baby some six weeks previously, was suffering so severely as to be incapacitated from nursing the child. Three, if not four, medical men had seen the patient, and the pains were as bad as ever. The treatment adopted had included the application of belladonna and chloroform liniments, friction with aconite ointment, the placing of first one arm and then the other in a sling, and the confining of the patient to bed. The liniments and ointments had done no good; the slings had given temporary relief; the confinement to bed was only useful so long as the patient refrained from sitting up and from holding the baby.

Mrs. A, age 30, is well known to the writer, although he had not seen her for some weeks prior to the time at which her husband spoke to him about her condition. That evenirg he saw her, and she informed him that the pain "shot" all over the arms, but was most intense over the lower part of the deltoid and the middle third of the biceps; it was aggravated by standing or on attempting to lift a weightfor example, the baby. Sleep, she said, was not much interfered with, as the pain passed off in about an hour after lying down.

In the course of conversation the following points were noticed: (1) The shoulders looked much less square than before the pregnancy; (2) the dress worn, which fitted accurately when it was new, some time early in the pregnancy, was wrinkled above the iliac crests, and at the back was rather dragged towards the axillx; (3) when sitting Mrs. A selected an armchair and sat leaning forward with her forearms resting on the arms of the chair and her shoulders pushed up towards her ears - a position not at all customary for her ; (4) after sitting in that position for some time she became markedly brighter and appeared to be relieved of the dragging pain.

The mental process leading up to the diagnosis was noted as follows:-

1. The area of maximum intensity of pain is the area of cutaneous distribution of the fifth cervical segment, and is not a single nerve area.

2. The pain is evidently of mechanical origin, because it is entirely relieved when the arms are supported or when the patient lies down, and is aggravated when the patient stands up or attempts to carry a weight upon her arms.

3. The shoulder girdle is held in position by the clavic!e, the muscles, and the convexity of the thoracic wall.

4. Durirg the later weeks of pregnancy the chest wall is dragged up by the diaphragm, which elevates the ribs into what is, in extreme cases, practically the full inspiratory position, with the result that the shoulder is raised. After childbirth the diaphragm descends and the ribs follow it down. Deprived of snpport the shoulders also sink. If the child be a large one these descents are to levels lower than those at which the parts lay before the pregnancy-a result of the relaxation of the abdominal musculature and the consequent descent of the abdominal viscera.

5. In the case of Mrs. A the shoulders are normally unusually square (male type). This is probably due to her athletic training as a girl at school. Her abdominal musculature must also, to judge by the physical feats of which she was capable, have been unusually well developed. During pregnancy the abdominal muscles could not possibly have been used as they were accustomed to be used; therefore they practically certainly lost much of their development. This, coupled with the facts decendent upon the size of the child, must have allowed an unusually great descent of the chest wall, and consequently of the shoulders, to occur after delivery.

6. When the shoulder girdle descends it travels round the arc of a circle of which the centre is at the sterno-clavicular articulation, and the radius the length of the clavicle. The nerves, however, must descend upon the arcs of circles, of which the ventres are at the transverse processes of their corresponding vertebræ, and the radii the lengths from these points to the points at which the highest branches are given cff to the muscles of the arm. In cases of marked descent of the shoulder girdle it is, therefore, a mechanical necessity that the rerves, more especially the fifth, are pulled upon, and pressed against the transverse processes of their own vertebræ. Such a condition of tension or pressure will be aggravated by the assumption of the erect attitude and by attempting to lift a weight, and will be diminished by supporting the arms or on lying down.

The diagnosis, therefore, was (1) that the pain in Mrs. A's arms was due to pressure of the fifth cervical nerves against the transverse processes of the fifth cervical vertebra; (2) that this was the result of a descent of the shoulder girdle consequent upon the descent of the ribs; (3) that the ribs descerded because they lost the support of the diaphragm which followed the viscera down when they gravitated toward the pelvis after childbirth; and (4) that the viscera descended further than is usual because of the atrophy which was superadded to the inevitable relazation of the abdominal muscles.

The immediate treatment suggested was to force up the diaphragm by allowing the weight of the abdominal viscera to come against it when the head and shoulders were depressed and the pelvis elevated, and then to apply some abdominal support (a folded towel placed inside the ordinary corset was used) before assuming the erect attitude. The ultimate treatment was to re-develop the abdominal muscles.

Asked about the condition at Easter, 1912-i.e., 18 months after the commencement of treatment-Mrs. A reported that the forcing and holding up of the diaphragm had from the very first entirely abolished the pain. Last summer rowing and tennis had re-developed the muscles, and all special precautions were abandoned. Once or twice this spring, when no efficient abdominal support has been applied and long days' tramping have been indulged in, there has been some pain in the arms in the afternoons when the abdominal muscles were getting tired, but this has at once disappeared on Mrs. A putting on a pair of corsets. The results seem to justify the diagnosis.

Dublin.

\section{CYSTITIS AND URINARY ANTISEPTICS.}

BY CAMPBELL WILLIAMS, F.R.C.S. ENG.

With a Note on Analyses of Crines for Formaldehyde by W. Harrison Martindile, Ph.D.

Hexamethylenetetramine and allied drugs are frequently prescribed in bacilluric cystitis and the early stages of gonorrhoea. A mixture containing helmitol $5 \mathrm{gr}$, potass. citratis $20 \mathrm{gr}$, ext. tritici repentis liq. $1 \mathrm{dr}$, syr. aurant. $\frac{1}{2} \mathrm{dr}$., aq. carui to $4 \mathrm{dr}$. is a favourite with me, the dose to be taken in an equal amount of water thrice or four times, the last dose at bedtime. This urinary antiseptic (helmitol) is stated to have the composition hexamethylenetetramine-anhydromethylene citrate, and it is by reason of its salt constitution incompatible with alkalies and their carbonates.

It is well known that the neutral citrate of potash is excreted as an alkaline carbonate, the citric radicle having been "burned up" in its passage to the bladder. One might imagine that the above amount of the potash salt taken in eight doses would reduce the ordinary acidity of normal urine, so that the tetramine compound would meet with either a neutral or an alkaline urine in the bladder. Although possibly it is split up into the base and the citrate salt, it would not be decomposed-arguing on analogy with hexamethylenetetramine-into formaldehyde. My clinical experience, however, led me to conclude that, whilst the combination of citrate of potash and helmitol reduced vesical irritability and gave relief to patients who had either the 
acid or bighly acid urine of bacilluria or the acidity so distressing in acute gonorrhoa, nevertheless the dosage was not sufficiently large as regards the potash salt to render the urine alkaline and so inhibit formic aldehyde liberation in the vesical or possibly the renal urine. The amount of formaldehyde set free might be reduced, but it would not be entirely non-existent. So long as the urine continued to show an acid reaction with litmus paper I continued with the citrate of potash.

The clinical results obtained determined me to persevere with my pharmaceutical heresy. But the letter of Dr. Anson Jordan, of Cambridge, in THE LANCET of March 9th, 1912 , p. 684, in connexion with Dr. David Newman's able lectures on cystitis and the employment of the aforementioned drugs in combination, ${ }^{1}$ led me to seek the aid of my friend, Dr. W. H. Martindale, in order to see whether I was relying on fact or fancy. He kindly undertook the analysis of samples of my own urine under conditions which are set forth in his report.

It is of interest to note that a small amount of formaldehyde was found in my urine under alkali (potassium citrate) treatment in the case of the over-night urine, but the analyst's conclusions may be read with regard to the matter. That none was detected in the morning urine may possibly be due to its dilute condition or the fact that it had been completely eliminated. It was pale straw-coloured, possibly owing to the best part of a pint of tea consumed at breakfast, in conjunction with a reduction in acidity which is presumed to occur normally at or about that time of day, constituting the so-called "alkaline tide."

I think that the results of the experiments tend to show that helmitol is not altogether wasted under the administration of potassium citrate, even when the drug is given in a case in which there is only normal urinary acidity, but potassium citrate should not be given in sufficient amount to render the urine quite alkaline. One might assume that the inhibition effects of the potash would be less in a case of bacilluric cystitis where the urine is at least irritatively acrid, even if it be not intensely so to the vesical mucous membrane.

\section{Note ON ANALYses OF SPECMIENS 13Y}

$$
\text { W. H. Martindale, PH.D. }
$$

Mr. Campbell Williams handed me two samples of his urine for examination. One of these had been passed overnightMarch 10th-and the other on the morning of March 11th at 10.45, un Jer treatment with 40 grains of helmitol and 160 grains of potassium citrate spread over 48 hours. The specimens were examined for the presence of formaldehyde.

The detection of small amounts of formaldehyde in urine is no simple matter. Preliminary experiments showed that tests commonly employed for detection of formaldehyde (when used as a preservative) in milk, \&c., yield unsatisfactory results in the case of urine. For example, Schiff's reagent yields a colouration with normal urines. Again, Rimini's test gives a greenish-brown (reddish-brown at first, quickly changing its colour); a small quantity of added formaldehyde is difficult to detect by this test in a normal urine. The phenol and sulphuric acid test is also unsatisfactory in view of the colour produced by the organic matter of the (normal) urine. The salicylic acid in sulphuric acid test of the U.S.P. is also not sufficiently delicate for the purpose.

The phloroglucin test (Extra Pharmacopcia, p. 890) was found to be more definite, as it gave no colour with normal urine, but in the presence of 1 part of formalin in 5000 of urine gave a decided terra-cotta or pinkish colour; there was a decided colouration with weaker strengths down to 1 in 50,000 which could be detected by comparing a blank test side by side. The evening sample of the urine showed presence of about 1 of formaldehyde in 50,000 by this reaction ( $=1$ of formalin in 20,000 ); the morning sample did not give any indication, but helmitol itself was found to give a perceptible colour with the phloroglucin test in proportion of 1 in 5000 of urine, though hardly any reaction with weaker strengths. An indication of formaldehyde was also obtained in the distillate from the evening sample by Rimini's test, but none was indicated by the morning specimen. Here, again, Schiff's reagent is useless, as three normal urines gave a positive result with the distillates, pointing to the presence of volatile aldehydes other than formaldehyde.

T The Lancer, Feb. 24th (p. 490) and March 2nd (p. 570), 1912.
It is important to note that both the samples of urine were distinctly acid, and, minute quantities of formaldehyde being found, it became a question whether more would have shown itself had drugs been administered purposely to render the urine acid. To determine this it was decided to conduct some experiments in vitro.

A volume of normal urine (200 c.c.), having acidity $=0.2$ per cent. $\mathrm{NaH}, \mathrm{PO}_{4}$, was divided into two equal parts.

One portion ( 1 ) was acidified by further 0.8 per cent. $\mathrm{NaH}_{2} \mathrm{PO}_{4}$ making 1 per cent. $\mathrm{NaH}_{2} \mathrm{PO}_{4}$, which may be deemed a maximum acidity for normal urine. To 30 c.c. of this was added $0.1 \mathrm{grm}$. helmitol it being argued that this proportion (i.e., a 15-grain dose in $\frac{1}{2}$ pint) might approximate the conditions in vivo-at any rate, the proportion would not be greater.

After 10 minutes at $40^{\circ} \mathrm{C}$. this liquid was tested as follows :-

10 c.c. with the phloroglucin test-distinct pink colour.

10 c.c. with the "meta" test (for details see below)-very slight opalescence in a few minutes, indicating possibly 1 of formalde hyde in between 5 and 10 thousand of urine.

After 10 hours at $40^{\circ} \mathrm{C}$. the liquid tested as follows:-

10 c.c. with phloroglucin-pinkish colour.

10 c.e. the "meta" test-copious precipitate developing in four minutes

30 c.c. of the other portion (B) treated with helmitol $0.1 \mathrm{grm}$. and then neutralised with sodium bicarbonate (without adding sodium acid phosphate) and tested as (A). gave as follows:-

After 10 minutes at $40^{\circ} \mathrm{C}$.

10 c.e. with the phloroglucin test-decided pink about same as $A$.

10 c.c. with the " meta" test-nil in four minutes.

After 10 hours at $40^{\circ} \mathrm{C}$.-

$10 \mathrm{c} . \mathrm{c}$. with the phioroglucin test-pinkish colour slightly less than with the acid series.

10 c.c. with the " meta" test-rery slight indication.

As control 30 c.c. of (B) were treated with 0.13 c.c. of formalin $(=0.05 \mathrm{grm}$. of formaldehyde). This amount of formaldehyde could be assumed to be vielded by $0.1 \mathrm{grm}$. of helmitol under the best conditions. (No allowance made for $\mathrm{H} . \mathrm{COH}$ in the methylene-citrate radicle-see No allowance
Conclusions.)

Atter 10 minutes at $40^{\circ} \mathrm{C}$.

10 c.c. with the phloroglucin test-crimson much stronger than (A) or $(B)$

i0 e.c. with the "meta" test-immediate opalescence and precipitate.

Ater 10 hours-

10 c.c. with the phloroglucin test-strong )

red colour

0 c.c. with the ". $\ldots, \ldots, \ldots \ldots \ldots, \ldots$, Much greater than precipitate

We know that the "meta" test shows 1 in 5000 at least of formaldehyde, and therefore as practically no reaction is seen either in the case of the acidified or neutral urines after 10 minutes at body temperature, and as we have here the opportunity of detecting about 1 in 570 of formaldeyde if decomposition of helmitol follows theory, it is clear that neither in acid nor in neutral urine has such complete decomposition taken place in that time. With regard to the 10-hour results, the "meta" test indicates more adequate production of formaldehyde in acid and little or none in neutral urine.

\section{Details of the " Meta" Test.}

In the course of this investigation $I$ arranged a test that will indicate 1 of formaldehyde in 10,000 of urine. A 5 per cent. solution of meta-diamido-benzol hydrochloride yields with even very ailute formaldehyde solution (in plain water) a jellowish colour. Working with Nessler glasses or test tubes on a white ground with controls this is clearly seen in a proportion of 1 in 5000, or indeed a 1 in 50,000 could be detected. The method employed is to add 0.5 c.c. of the solution to 10 c.c. of liquid to be tested; the yellowish colour develops in about three to four minutes and is very characteristic. With urines the following facts were determined:-

10 c.e. of normal urine treated with " meta" shows no colour change. 10 c.c. of normal urine containing helmitol in proportion of $]$ in 5000 gives no change.

10 c.c. of normal urine containing 1 of formaldehyde in 5000 gives an opalescence and ultimate bulky precipitate, developing in a few minutes.

The reaction was easily seen in an alkaline urine to which formaldehyde was added in proportion 1 in 10,000 .

The reaction is of interest - I have not seen it described elsewhere-and is possibly explained by assuming a condensation between the "meta" and formaldehyde, with the production of a substance which interacts with some constituent or constituents of normal urine. A 3 per cent. urea solution was found to produce with 1 in 5000 formaldehyde a similar precipitate, but more slowly (a matter of two hours). The exact explanation of the test at the time of writing has not been determined. It might be of use in the examination of urine for detecting formaldehyde poisoning which sometimes occurs. 
Examination of Urine under Helmitol and Dilute Nitro. hydroohlorio Aoid Treatment.

It may be added that the "patient" underwent a selfinflicted régime of acid. nitro-hydrochlor. dil. with helmitol alternately. He took two $15 \mathrm{~m}$. doses of the former at respectively 12 midday and 8 P.M. and two 5 grain doses of helmitol at 2 and 10 P.M. respectively on March 12th. The overnight urine, 35 ounces, and the next morning's were examined. It is well-known that the administration of mineral acids does not necessarily increase acidity of the urine; at the same time, propter hoc or otherwise, there was relative increased acidity and vesical irritability.

The "meta" test in the overnight specimen showed formaldehyde less than 1 in 5000, whilst the morning's urine also showed a minnte, practically negligible, amount. This urine was then "helmitolised" and "formalised" respectively and tested as before, with result that-

1 of helmitol in 10,000 gave (as anticipated) nil.

1 of formaldehyde in 10,000 gave characteristic opalescence and

precipitate.

The examinations of Mr. Campbell Williams's nrine showed whilst under alkali treatment an amount of formaldehyde which, though minute, was doubtless commensurate with the doses taken. On the other hand, the in vitro experiments after the lapse of ten hours showed larger yield of formaldehyde under sodium acid phosphate than the experiments with neutral urine-the ten-minute results, however, being practically identical. In other words, Mr. Campbell Williams's claim was substantiated so far as the experiments went in oorpore vili, but in the method adopted to simulate the process in the body there were signs of more marked formaldehyde production in the acidified urine.

It is open to discussion whether the oral administration of sodium acid phosphate or other acid-rendering substances produces so marked an effect on the urine as in the laboratory experiment. It would seem that the formaldehyde liberation is probably not greater under acid treatment than under potassium citrate. (It may be remarked that it is claimed that the substance acts equally well as an antiseptic whether in acid or alkaline urine. It should also be clearly understood that the anhydromethylene citrate radicle liberates formaldehyde, and probably has greater tendency to do so than hexamethylenetetramine itself.)

The conditions in the case of hexamethylenetetramine are somewhat different.

\section{Hexamethylenetetramine Experiments.}

It was thought of interest to carry out an investigation with hexamethylenetetramine on identical lines with the foregoing in vitro experiments:-

Two portions of urine were treated exactiy as in the helmitol experiments, but with hexamethylentetramine in place of the helmitol. Kesults were as follows:-

(A) Acidified :-

10 minutes-

Phloroglucin test--slight pink.

"Meta" test-minute or $n i$.

10 hours-

Phloroglucin-slight pink, possibly 1 in 50,000

" Meta" test-practically nil (when carried to 20 hours slight precipitate).

(B) Neutralised :-

10 minutes-

Phloroglucin test-slight pink (shade less than A).

"Meta" test-minute or nil.

10 hours-

Phloroglucin test--practically nil.

"Meta test"-practically nil.

Control see the Control under helmitol experiments.

Conolusion. - There was no decomposition in either neutral urine or urine acidified with acid sodium phosphate in 10 minutes, but in 20 hours there was evidence of slight formaldehyde formation in urine so acidified. This would indicate that to obtain formaldehyde liberation from hexamethylenetetramine the simultaneous administration of an acid rendering substance is necessary, and per contra the compound should not be given with drugs in sufficient quantity to produce an alkaline urine. The following view of the action of hexamethylenetetramine is taken from the United States Dispensatory.

Taken internally it is eliminated with great rapidity generally unchanged, its presence often being detected in the urine in half an hour, and as long as 24 hours, after its ingestion. The old belief that
it was largely decomposed with the liberation of formaldehyde in the kidney is probably not correct, although frequently formaldehyde may be detected in the urine after its administration. The change appears to take place after excretion from the kidney, and probably occurs only when there is an excess of acid in the urine. Hexamethylenetetramine probably is itself possessed of marked bactericidal powers, and it probably achieres good in urinary diseases by virtue of its ${ }_{2}$ own inherent properties.

\section{THE TREATMENT OF CANCER IVITH SELENIUM.}

BY CHARLES E. WALKER, D.SC. LIVERP., M.R.C.S. ENG, , L.R.O.P. Lovd.

(From the Researoh Department, Glasgow Caneer Hospital.)

THE announcement that by the intravenous injection of a "compound of selenium and eosin" Professor Wassermann had been able to cure small cancerous tumours produced in mice by inoculation, ${ }^{1}$ has no doubt led to many experiments with varions preparations of selenium. Professor Wassermann gives no indication as to the nature of the preparation used by him, but a true compound of selenium and eosin must be of extreme complexity. A definite claim to the successful treatment of cancer in the human subject by intravenous injection of selenium in the colloidal form has been made recently. ${ }^{2}$

In view of these facts it may be of interest to the members of the medical profession to have an account of experiments with preparations of selenium carried out in a laboratory where great facilities exist. Almost immediately after the publication of Professor Wassermann's results, with the assistance of Dr. H. E. Whittingham and Mr. F. J. Robinson, I began experiments with selenium. The first preparation tried was colloidal selenium and eosin, which apparently form a colloidal complex. ${ }^{3}$ These experiments are of particular interest in view of the claims now made for colloidal selenium. It has been suggested that chemically prepared colloidal selenium is not in sufficiently finely divided particles, and that resort has been had to the electric arc, as is the case in the preparation of the colloidal forms of the metals. ${ }^{4}$ It is difficult to see how this has been possible as selenium is a very effective non-conductor. Its conductivity is increased, or rather its non-conductivity is decreased, by a very strong light, but the production of an electric arc in distilled water between points of selenium would appear to be so difficult as to be almost impracticable. The satisfactory preparation of selenium for therapeutical purposes in the colloidal form by chemical methods is sufficiently difficult. It is easy enough to arrive at the colloidal condition, but the difficulty is to obtain a permanent preparation. The colloidal selenium tends, upon the slightest provocation, to precipitate in the red amorphous form or to turn into the dioxide $\left(\mathrm{SeO}_{2}\right)$.

With experiments extending over two montbs sufficient facts were added to those already available to obtain a permanent preparation. The statement that the particles are not sufficiently small except when the electrical method is resorted to, if any electrical method has been discovered, does not apply to the preparations made in this laboratory, and the colloid is just as active at the end of six weeks as on the day it was made, and is not precipitating. Eosin is supposed to be an electrolyte to colloidal selenium. Practically the eosin used here has not so acted.

Mice and rats with grafted malignant growths of various sizes have been absolutely unaffected by intravenous injections of this preparation except that they have turned pink within 30 seconds or a minute of the injection, and their excreta have been pink for two or three days. Wassermann's preparation was highly toxic. The colloidal selenium, with or without eosin, is non-toxic. I have injected 0.25 c.c. into mice and 1 c.c. into rats without any ill-effects. It must be realised in this connexion that $0.25 \mathrm{c}$. c. In a mouse corresponds roughly to 700 c.c. in a man. No effect what ever was produced upon the tumours in either mice or rats. I have found that colloidal selenium is not toxic to monkeys, and the French reports state that it is not toxic to man. On

1 The Laxcet, Jan. 13th, 1912, p. 112 ; Jan. 27 th, p. 264. 2 Sociét Medicale des Hupitaux de Paris, Feb. 17 th and March 1 st and Brit. Hed. Jour., April 13th.

3 The reasons for this conclusion are ton technical to be of general interest.

\pm Brit. Ned. Jour, April 13th. 